\title{
Recurrent Primary Laryngeal Amyloidosis in a 36 Year-Old-Woman
}

\author{
Srujitha Murukutla a, d, Vijaya Raj Bhatt ${ }^{\mathrm{a}}$, Abhirami Vivekanandarajah ${ }^{\mathrm{b}}$, \\ Michael J Pitman ${ }^{c}$, Meekoo Dhar ${ }^{\mathrm{b}}$
}

\begin{abstract}
Laryngeal amyloidosis, a rare form of primary amyloidosis, is often benign and runs an indolent course. It will often recur, necessitating regular follow up to detect local recurrence as well as development of systemic amyloidosis. We report a case of a middle-aged woman with recurrent laryngeal amyloidosis and discuss relevant literature.
\end{abstract}

Keywords: Laryngeal; Amyloidosis; Recurrent

\section{Introduction}

Amyloidosis, characterized by extracellular deposition of amorphous proteinaceous material in various organs, can be systemic or localized. In laryngeal amyloidosis, a rare entity, surgical treatment can be curative but it often recurs [1]. We report a case of a recurrent laryngeal amyloidosis in a 36-year-old woman.

\section{Case Report}

A 36-year-old woman was referred to the ambulatory oncol-

Manuscript accepted for publication March 9, 2012

${ }^{a}$ Staten Island University Hospital, Department of Medicine, 475

Seaview Avenue, Staten Island, New York, USA

${ }^{\mathrm{b}}$ Sanford R Nalitt Institute for Cancer and Blood Related Diseases,

Staten Island University Hospital, Division of Hematology and

Oncology, Department of Medicine, 256 Mason Avenue, Staten Island,

New York, USA

${ }^{c}$ Voice and Swallowing Institute, Department of Otolaryngology, New

York Eye and Ear Infirmary, New York, USA

${ }^{\mathrm{d}}$ Corresponding author: Srujitha Murukutla, Department Of Internal Medicine, Staten Island University Hospital, 475 Seaview Avenue, Staten Island, NY, 10305, USA. Email: srujitha.murukutla@gmail.com

doi: http://dx.doi.org/10.4021/jmc636e ogy clinic by her otolaryngologist. The patient was completely asymptomatic 3 years ago when she started complaining of gradual and progressive hoarseness of voice. She denied any shortness of breath, stridor, difficulty in swallowing, fever, night sweats, skin rash, bleeding from any site, fatigue, bone pain, numbness or weakness. Her medical and surgical history was significant only for laser therapy for a retinal tear involving left eye. Her medication included oral contraceptive pills only. Family history was significant for gastric cancer in father and hypertension in mother. She did not have any history of smoking or drinking.

An otolaryngologist had evaluated the patient for the hoarseness of voice. The entire head and neck examination was normal except for laryngeal findings. A laryngovideostroboscopic examination revealed irregular submucosal nodularity of the ventricles and vestibular folds as well as inflammation of the left vocal fold (Fig. 1). Blood chemistry, hemogram, chest $\mathrm{x}$-ray, and electrocardiogram were normal. A contrast-enhanced computed tomography (CT) of the neck revealed benign appearing nodularity of the anterior supraglottis. The patient underwent direct laryngoscopy under general anesthesia with carbon dioxide laser excision of all the involved supraglottic tissue. The left vocal fold, though it appeared to be mildly infiltrated by amyloid, was spared to preserve optimal phonatory function (Fig. 2). The biopsy

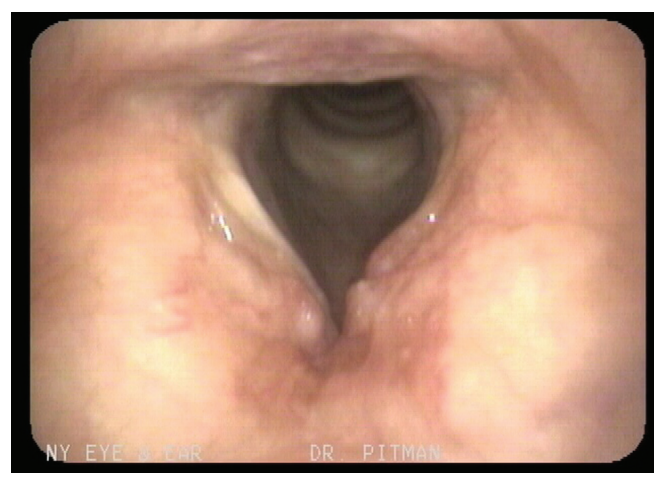

Figure 1. Image of the patient's original laryngovideostroboscopy. Note the smooth submucosal nodularity of the anterior vestibular folds and ventricles as well as inflammation of the left vocal fold due to amyloid infiltration. 


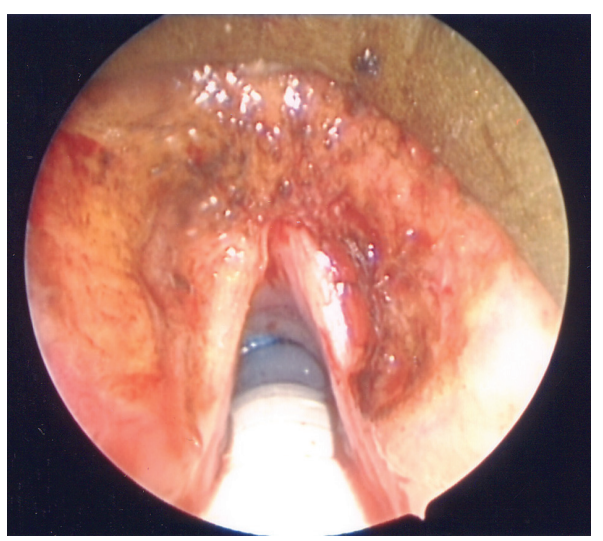

Figure 2. Intra-operative image status post excision. Note complete excision of supraglottic amyloid with preservation of the left vocal fold despite signs of likely amyloid infiltration of the left anterior vocal fold.

showed mild chronic laryngitis with protein deposition and no evidence of monoclonal cells. Protein deposits showed apple green birefringence under polarized light consistent with laryngeal amyloidosis (Fig. 3). The patient was instructed to follow-up with an oncologist for further work-up.

On evaluation in the oncology clinic, she had blood pressure of $140 / 73 \mathrm{mmHg}$, pulse rate of $76 / \mathrm{min}$, respiratory rate of $14 / \mathrm{min}$ and temperature of $36.4{ }^{\circ} \mathrm{C}$. There was no pallor, icterus, edema, skin rash, palpable lymph node, or macroglossia. Cardiac examination revealed absence of jugular venous distension or palpable thrill; cardiac apex was not displaced and normal heart sounds without any added sounds were heard. Abdominal examination did not reveal any organomegaly. There was no evidence of any neuropathy. Rest of the examination was also unremarkable.

Laboratory investigations showed normal hemogram, electrolytes, coagulation profile, urine analysis as well as liver and renal function tests. Urine and serum electrophoresis did not reveal any monoclonal protein. Computed Tomography (CT) chest, abdomen and pelvis and electrocardiogram were unremarkable; echocardiogram did not reveal any evidence of cardiomyopathy.

The patient had already undergone resection; hence she was instructed to follow up with the otolaryngologist on a regular basis.

Two years later, she returned to our clinic complaining of the recurrence of hoarseness of voice. She denied any other symptom. Repeat work-up for systemic amyloidosis was negative. Laryngovideostrobscopy performed by her otolaryngologist revealed a recurrence of the supraglottic nodularity and it was presumed the amyloid had recurred. The lesion was again excised via direct laryngoscopy using carbon dioxide laser. Following the excision, the patient had return of normal phonatory function and continues to do well 5-months status post her second surgery.

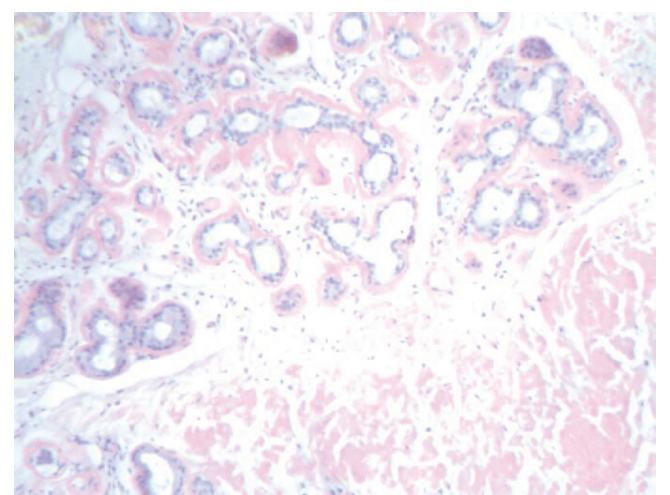

Figure 3. Congo red stain shows red staining for amyloid in the periglandular stroma, surrounding stroma and blood vessel endothelium.

\section{Discussion}

Laryngeal amyloidosis, the most common site involved in the head and neck region [2], is very rare and accounts for $<$ $1 \%$ benign laryngeal tumors [3]. Although usually primary and localized, it can also be a part of systemic disease, or secondary to an underlying disease or malignancy $[1,4,5]$. The pathogenesis of localized amyloidosis is not clear but is thought to be secondary to the local production of abnormal protein by plasma cells. Positive immunohistochemistry staining for plasma cells and light chains in the amyloid deposit supports the diagnosis. Thus, primary amyloidosis can be considered as a type of benign plasma cell dyscrasia [2]. The presence of local lymphoplasmacytic infiltration in laryngeal amyloidosis is distinct from systemic amyloidosis where the amyloid deposition and the plasma cells occur at different places [1].

Laryngeal amyloidosis is more common in men and at an old age with the mean age at diagnosis of 60 years [6]. Common sites involved include the following (in the descending order of involvement): true vocal cords, ventricles, false vocal cords, aryepiglottic folds and subglottic space [7]. Laryngeal amyloidosis can also be a part of multifocal disease with simultaneous involvement of other non-laryngeal sites $[1,8]$. The most common presenting feature is hoarseness of voice [6]. Patients can also present with dyspnea [9] and rarely dysphagia [10] or stridor [11]. Laryngoscopic examination often reveals nodular lesions or sub-mucosal edema [12]. Direct laryngoscopy and excisional biopsy of the lesion, as in our patient, is diagnostic, excludes laryngeal malignancy and can be therapeutic as well.

Histopathological examination with haematoxylin and eosin shows eosinophilic amorphous proteinaceous material; congo red staining shows apple green birefringence under polarized light [13]. Small cell carcinoma of the larynx and invasive medullary carcinoma of the thyroid are rarely associated and need to be ruled out [1]. In a patient with laryn- 
geal amyloidosis, it is also important to rule out systemic and secondary forms of amyloidosis [8]. Complete blood count, liver and renal function tests, urine and serum electrophoresis, electrocardiogram and echocardiogram are necessary. If warranted, abdominal fat pad biopsy may be necessary to exclude systemic involvement [2].

Microlaryngoscopy with carbon dioxide laser excision preserves laryngeal functions and remains the mainstay of therapy for primary localized laryngeal amyloidosis [2]. For localized amyloidosis at other sites, surgical intervention can be required particularly in the presence of complications such as hemorrhage, stenosis or obstruction [14]. Organ and disease-specific interventions including chemotherapy are indicated in systemic or secondary cases [15]. Prognosis of primary laryngeal amyloidosis, unlike other forms, is excellent with patients dying with disease, rather than due to the complications of the disease [1]. Recurrence, however, is likely to occur, thus regular follow up with laryngoscopy is imperative $[1,2,12]$. The patient should also be monitored for the development of systemic amyloidosis [1]. Recurrence occurs often secondary to the difficulty of performing a complete resection while preserving vital structures. Residual disease is frequently intentionally left to preserve laryngeal function. The duration of follow up remains unknown.

\section{Conclusion}

1) Laryngeal amyloidosis is rare and usually represents a form of primary amyloidosis. However, systemic amyloidosis and secondary forms need to be excluded; 2) Direct laryngoscopy and excisional biopsy of the lesion along with histopathological examination is diagnostic, excludes laryngeal malignancy and can be therapeutic as well; 3) Hematoxylin and Eosin staining shows eosinophilic amorphous proteinaceous material whereas congo red staining shows apple green birefringence under polarized light. These findings clinch the diagnosis; 4) Long-term follow-up is often necessary to monitor for disease recurrence and the development of systemic amyloidosis.

\section{Conflict of Interest}

None.

\section{Source of Funding/Grants}

None.

\section{References}

1. Thompson LD, Derringer GA, Wenig BM. Amyloidosis of the larynx: a clinicopathologic study of 11 cases. Mod Pathol. 2000;13(5):528-535.

2. Ma L, Bandarchi B, Sasaki C, Levine S, Choi Y. Primary localized laryngeal amyloidosis: report of 3 cases with long-term follow-up and review of the literature. Arch Pathol Lab Med. 2005;129(2):215-218.

3. Hellquist H, Olofsson J, Sokjer H, Odkvist LM. Amyloidosis of the larynx. Acta Otolaryngol. 1979;88(56):443-450.

4. Velez D, Hinojar-Gutierrez A, Nam-Cha S, AcevedoBarbera A. Laryngeal plasmacytoma presenting as amyloid tumour: a case report. Eur Arch Otorhinolaryngol. 2007;264(8):959-961.

5. Kojima M, Sugihara S, Iijima M, Ono T, Yoshizumi T, Masawa N. Marginal zone B-cell lymphoma of minor salivary gland representing tumor-forming amyloidosis of the oral cavity. A case report. J Oral Pathol Med. 2006;35(5):314-316.

6. Biewend ML, Menke DM, Calamia KT. The spectrum of localized amyloidosis: a case series of 20 patients and review of the literature. Amyloid. 2006;13(3):135142.

7. Tammam NH. Laryngeal Amyloidosis. Bahrain Medical Bulletin. 2005;27(no 3).

8. Passerotti GH, Caniello M, Hachiya A, Santoro PP, Imamura R, Tsuji DH. Multiple-sited amyloidosis in the upper aerodigestive tract: case report and literature review. Braz J Otorhinolaryngol. 2008;74(3):462466.

9. Siddachari RC, Chaukar DA, Pramesh CS, Naresh KN, de Souza CE, Dcruz AK. Laryngeal amyloidosis. J Otolaryngol. 2005;34(1):60-63.

10. Yiotakis I, Georgolios A, Charalabopoulos A, Hatzipantelis P, Golias C, Charalabopoulos K, Manolopoulos L. Primary localized laryngeal amyloidosis presenting with hoarseness and dysphagia: a case report. J Med Case Reports. 2009;3:9049.

11. Tsai TL, Chu PY, Chang SY. Laryngeal amyloidosis with airway obstruction. Otolaryngol Head Neck Surg. 2002;126(3):329-330.

12. Pribitkin E, Friedman O, O'Hara B, Cunnane MF, Levi D, Rosen M, Keane WM, et al. Amyloidosis of the upper aerodigestive tract. Laryngoscope. 2003;113(12):20952101.

13. Pang KP, Chee LW, Busmanis I. Amyloidoma of the nose in a pediatric patient: a case report. Am J Otolaryngol. 2001;22(2):138-141.

14. Bedioui H, Chebbi F, Ayadi S, Fteriche F, Sassi K, Jouini $\mathrm{M}$, Ksantini R, et al. [Gastric amyloidosis mimicking malignancy. A case report]. Ann Chir. 2006;131(8):455458.

15. Merlini G, Seldin DC, Gertz MA. Amyloidosis: pathogenesis and new therapeutic options. J Clin Oncol. 2011;29(14):1924-1933. 